\title{
1 Variation and constraints in hybrid genome formation
}

2

3 Anna Runemark $^{1}$, , Cassandra N. Trier ${ }^{1}$, Fabrice Eroukhmanoff ${ }^{1}$, Jo S . Hermansen ${ }^{1}$,

4 Michael Matschiner $^{1}$, Mark Ravinet ${ }^{1}$, Tore O. Elgvin ${ }^{1}$, and Glenn-Peter Sætre ${ }^{1}$

5

6 Affiliations:

$7 \quad{ }^{1}$ Department of Biosciences, Centre for Ecological and Evolutionary Synthesis,

8 University of Oslo, PO Box 1066, N-0316, Oslo, Norway.

9

$10 *$ Corresponding author. Email: anna.runemark@ibv.uio.no (A.R.). 


\section{Summary}

Recent genomic investigations have revealed hybridization to be an important source of variation, the working material of natural selection ${ }^{1,2}$. Hybridization can spur adaptive radiations $\mathbf{3}^{3}$, transfer adaptive variation across species boundaries $^{4}$, and generate species with novel niches ${ }^{5}$. Yet, the limits to viable hybrid genome formation are poorly understood. Here we investigated to what extent hybrid genomes are free to evolve or whether they are restricted to a specific combination of parental alleles by sequencing the genomes of four isolated island populations of the homoploid hybrid Italian sparrow Passer italiae $^{6,7}$. Based on 61 Italian sparrow genomes from Crete, Corsica, Sicily and Malta, and 10 genomes of each of the parent species $P$. domesticus and $P$. hispaniolensis, we report that a variety of novel and fully functional hybrid genomic combinations have arisen on the different islands, with differentiation in candidate genes for beak shape and plumage colour. There are limits to successful genome fusion, however, as certain genomic regions are invariably inherited from the same parent species. These regions are overrepresented on the Z-chromosome and harbour candidate incompatibility loci, including DNArepair and mito-nuclear genes; loci that may drive the general reduction of introgression on sex chromosomes ${ }^{8}$. Our findings demonstrate that hybridization is a potent process for generating novel variation, but variation is limited by DNA-repair and mito-nuclear genes, which play an important role in reproductive isolation and thus contribute to speciation. 
36 Introgressive hybridization can transfer adaptive genetic variation across species

37 boundaries $^{4}$ and generate new species ${ }^{2,4,5}$. Although historically thought to be

38 unimportant in animals, the genomic revolution has shown hybridization to be a

39 pervasive evolutionary force, common in both plants and animals, ${ }^{1,2}$ and one that has

40 even shaped the genome of our own species ${ }^{8}$. Portions of the genome vary in extent of

41 introgression ${ }^{4,9}$, and homoploid hybrid genomes are predicted to have unequal

42 parental contributions ${ }^{10}$. However, how hybridization, and subsequent recombination

43 and selection mold genomes is not well understood. For instance, it is not known what

44 determines the relative contribution of each parent and whether the genomic locations

45 of introgression are subject to constraints or are largely stochastic. Moreover,

46 genomic constraints in hybridizing taxa can be used to identify genes involved in

reproductive isolation between species as they do not introgress ${ }^{7}$, and hence are

important for our understanding of speciation and how biodiversity arises. One well-

supported finding is reduced introgression on sex chromosomes, which is common in

species where one sex is heterogametic ${ }^{8,11}$. To what extent this pattern is caused by

51 specific types of genes on sex chromosomes is debated ${ }^{12}$, and knowledge of the genes causing incompatibilities and reproductive isolation is needed to resolve this.

54 To investigate whether certain genes must be inherited from a specific parent species

55 to form a stable and functional hybrid genome and whether divergent genomes can

56 arise from hybridization, genome-wide data from multiple independent hybrid

57 populations are required. We studied isolated island populations of the homoploid

58 hybrid Italian sparrow Passer italiae ${ }^{6,7}$ (Fig. 1a) to determine the constraints on, and

59 variability of, genome regions and gene categories. The Italian sparrow genome is a

60 mosaic of the genomes of its parent species, the house $P$. domesticus and Spanish $P$. 
61 hispaniolensis ${ }^{13}$ sparrows. The species is thought to have originated when house sparrows colonized the Mediterranean less than 10,000 years ago ${ }^{6,14}$. Morphologically

63 divergent populations of Italian sparrows are found on the Mediterranean islands of

64 Crete, Corsica, Sicily and Malta $\left(\right.$ Fig $\left.1 \mathrm{a} ;{ }^{15}\right)$ and we used these isolated island

65 populations to investigate genome-wide patterns of divergence and differentiation

66 from the parent species and within the hybrid species (Supplementary Tables 1-2).

67 We sequenced 10-21 Italian sparrow genomes from each island and 10 genomes from

68 each of the parent species to 6-16X coverage as well as a tree sparrow (P. montanus)

69 as an outgroup and aligned them to the recently de novo assembled house sparrow

70 reference genome ${ }^{13}$.

72 To determine if these Italian sparrow island populations were genomically

73 differentiated, we first used a principal component analysis. Our results show strong

74 support for differentiation among the hybrid island populations and from the parent

75 species (Fig 1b; Supplementary Fig. 1; Supplementary Tables 3-4). The Italian

76 sparrow populations differ in position along the axis of divergence of the parent

77 species, with Crete and Corsica closer to house sparrow and Sicily and Malta closer to

78 Spanish sparrow (Supplementary Table 5). To further investigate population

79 differentiation, we assessed the most likely number of genetic clusters in the data, and

80 the individual probability of belonging to these clusters using a structure analysis. We

81 found support for two clusters (Supplementary Table 6) corresponding to the parent

82 species and intermediate assignment probabilities for the Italian populations, with

83 Crete and Corsica having the highest probabilities of clustering with house sparrow

84 and Sicily and Malta the highest probabilities of clustering with Spanish sparrow (Fig.

85 1c). The assignment probabilities differed significantly among populations (ANOVA 
$86 \quad \mathrm{~F}_{3,47}=736.54, P=2.2 \mathrm{e}-16$; Supplementary Table 7), and across the genome

87 (Supplementary Fig. 2). Moreover, in the most frequent phylogenetic tree topology,

88 house sparrow, Crete and Corsica form one cluster and Spanish sparrow, Malta and

89 Sicily the other, with Crete and Malta clustering most closely with parent species

90 (Fig. 1d and Supplementary Fig. 3). The phylogenetic clustering varied spatially over

91 the genome (Fig. 1e; Supplementary Table 8). We also found significantly higher

92 Spanish sparrow introgression in the Sicily and Malta populations (Patterson's D:

$93 \quad$ ANOVA $_{3,115}=22.52, P<0.001$; Supplementary Table 9). The non-recombining

94 mitochondrial DNA was similar to that of house sparrow for all Italian sparrows, with

95 the exception of one Corsican individual having Spanish sparrow mitochondrial

96 DNA, and two Maltese individuals appearing to have both house and Spanish sparrow

97 mitochondria (Fig. 1f). This is consistent with heteroplasmy, as previously reported

98 for mainland Italian sparrows ${ }^{13}$.

99

100

101

102

103

104

105

106

107

108

109

110

To address whether differentiation within a hybrid species is likely to result from positive selection or if differentiated areas mainly arise as a by-product of long-term linked background selection in low recombination areas ${ }^{16}$, we first investigated whether differentiation among Italian populations correlates with that between the parent species. We then tested whether variation in differentiation reflects variation in recombination rate, as has previously been shown in flycatchers ${ }^{16}$. Differentiation among the Italian sparrow populations was not strongly correlated with differentiation between the parents, (Supplementary Fig. 4). The standardized slope (beta) of the relationship between within-Italian differentiation and recombination rate was much shallower than that between the parent species differentiation and recombination rate

10 (Supplementary Fig. 4; Tables S10-S11), and we therefore find no strong evidence for 
111 house sparrow recombination rate accounting for the genomic heterogeneity observed

112 within this hybrid species. Sorting of parental variants, potentially due to Hill-

113 Robertson effects ${ }^{17}$, may have contributed to this reduction in differentiation in low

114 recombination regions compared to that of the parent species. Regions with high

115 differentiation may instead reflect divergent selection, or, alternatively, the

116 recombination landscape may be altered following hybridization and spur

117 differentiation in other areas of the genome in the hybrid than in its parents.

119 To identify regions under divergent selection in the hybrid Italian sparrow we

120 extracted the windows where the island populations were most divergent (measured

121 as relative similarity to the parent species in terms of $F_{\mathrm{ST}}$ ). Among the genes found in

122 the $1 \%$ windows that are most divergent between the island populations, 43 different

123 gene ontology $(\mathrm{GO})$ categories were overrepresented relative to the rest of the

124 genome (Supplementary Table 12). Among these categories were genes related to

125 neuron function, including nervous system development, transmission of nerve

126 impulse, and synaptic transmission (Supplementary Table 12), suggesting that these

127 categories of genes have been under divergent selection. Interestingly, genes related

128 to neuron function have also been targets of recent positive selection in great tits ${ }^{18}$.

129 The outliers also included FGF10, a gene explaining beak shape divergence between

130 Darwin's finches ${ }^{19}$. Sicily and Crete were strongly divergent at this beak shape

131 candidate gene and the Italian sparrow has previously been shown to exhibit adaptive

132 beak shape divergence ${ }^{20}$ (Fig. 2a-c). A gene involved in feather development ${ }^{21}$ and

133 melanogenesis ${ }^{22}$, wnt7A, was also a highly differentiated outliers among the

134 otherwise genetically very similar (mean $\left.F_{\mathrm{ST}}=0.016\right)$ but plumage-wise divergent

135 Sicilian and Maltese populations ${ }^{15}$ (Fig. 2d-f and Supplementary Table 12). These two 
examples underscore that repeated hybridization between the same parental species can generate locally adapted populations through reshuffling of parental alleles at

138 biologically important loci.

140 To identify areas of unique Italian sparrow evolution, we targeted regions in which

141 the Italian sparrow populations have diverged from both parents by extracting the $1 \%$

142 of windows exhibiting the largest differences in $F_{\mathrm{ST}}$ between each Italian/parent

143 comparison, only keeping windows overlapping between hybrid/parent in both

144 comparisons. We found 12 overrepresented GO categories (Supplementary Table 13)

145 including circadian rhythm, entrainment of circadian clock and rhythmic process:

146 these genes showed strong signals of stabilizing selection (Fig. 2g), and elevated

147 linkage disequilibrium (Supplementary Table 14). These results illustrate how

148 population specific selection in concert with the parental mosaic is able to form

149 unique features in the genomes of hybrid populations.

152 The level of differentiation between the Italian sparrow and the parent species is

153 elevated on the Z-chromosome compared to autosomes (Paired t-test; $\mathrm{t}_{3}=-8.40$

$154 P=0.004$; Supplementary Fig. 5-6). This is expected based on the lower effective

155 population size of this sex chromosome and hence elevated rates of genetic $\mathrm{drift}^{23}$.

156 However, increased differentiation on the Z-chromosome is also expected from the

157 faster $\mathrm{X}(\mathrm{Z})$ effect of elevated rates of adaptive evolution on the macro sex

158 chromosome due to hemizygous exposure ${ }^{24}$. Outlier loci are strongly overrepresented

159 on the Z-chromosome (Fig. 2h; all $P$ 's $<0.001$; Supplementary Table 15), except for

160 the outliers in the category where Italian populations invariably had inherited Spanish 
161 sparrow alleles, none of which resided on the Z. Interestingly, Tajima's D estimates

162 for the Z-chromosome have significantly higher variance than those for the autosomes

163 (Fig 2c; Repeated measures ANOVA F ${ }_{1,3}=56.94 ; P=0.005$; Supplementary Fig. 7-8)

164 and dn/ds was higher on the Z-chromosome compared to autosomes (goodness of fit

$165 P<0.001$ for fixed differences against both house and Spanish sparrows;

166 Supplementary Table 16), supporting a role for selection driving strong Z-

167 chromosome divergence.

169 Across taxa with heteromorphic sex chromosomes, introgression on sex chromosomes

170 is reduced ${ }^{8,11}$. To detect loci potentially important in causing such reduction in

171 introgression on the sex chromosomes, we identified regions invariably inherited from

172 a specific parent across all populations. We summed the $F_{\mathrm{ST}}$ against house sparrow

173 across island populations and subtracted the summed $F_{\mathrm{ST}}$ against Spanish sparrow

174 before extracting the extremes at both ends of the distribution (the $2 \%$ of the windows

175 with squared values most diverged from 0; Supplementary Table 17). We found

176 strong evidence for genes invariably inherited from house sparrow, especially on the

177 Z-chromosome. DNA damage stimuli were significantly overrepresented among these

178 genes $\left(P_{\text {DNArepair }}=0.026\right)$. There were 11 mitonuclear loci and although these were not

179 generally overrepresented $\left(P_{\text {mitonuclear }}=0.11\right), 7$ were found in the areas on the Z-

180 chromosome strongly constrained to house sparrow inheritance, including the

181 previously identified candidate incompatibility gene HSDL2 ${ }^{7}$ (Supplementary Table

182 18; Fig. 3). There were also 6 DNA mismatch-repair genes on the Z-chromosome,

183 among them the candidate incompatibility gene GTF2 $\mathrm{H} 2^{7}$ which is involved in

184 nucleotide excision repair (Fig. 3). This suggests that hybrid genome formation is

185 restricted to uniparental inheritance for these gene classes. 
187 Whereas mito-nuclear genes are known as drivers of reproductive isolation ${ }^{7}$ and are 188 expected to be under selection to interact with the frequent house sparrow-like 189 mitochondria, the role for DNA repair genes is less established. However, reduced 190 DNA repair functioning ${ }^{25}$ has been found in Xiphophorus fish hybrids, and the 191 mismatch repair systems have been shown to contribute to meiotic sterility and cause 192 incompatibilities in yeast ${ }^{26}$. Hence, multigenic DNA repair pathways may need parent 193 specific inheritance to function. As most of these outlier genes were located on the Z194 chromosome, they may contribute to the pattern of reduced introgression on sex 195 chromosomes ${ }^{7}$.

197 Our comparison of isolated homoploid hybrid populations formed from the same parent species combination reveals that hybridization can produce diverged genomes 199 with a range of different proportions of parental contribution. More outlier genes 200 exhibited invariable inheritance from house sparrows than from Spanish sparrows. 201 This suggests that parts of the genome must be inherited exclusively from one of the 202 parent species, while the rest of the genome may vary with respect to parent species 203 inheritance. Purging of Dobzhansky-Muller incompatibilities ${ }^{27}$ has been suggested to 204 be important for shaping hybrid genomes. We find that house sparrow DNA-repair 205 genes and mito-nuclear genes are necessary for "escaping the mass of unfit 206 recombinants" ${ }^{28}$, most likely due to epistatic interactions. Genes invariably inherited 207 from the Spanish sparrow are fewer but include a candidate pigmentation gene $208 \mathrm{WNT}^{29}$ and a gene involved in vision, OLFML2 $\mathrm{B}^{30}$, hence affecting external 209 phenotype rather than genome function. Hence, both genome and organismal function 
210 can constrain hybrid genome formation, and the relative importance of the two may

211 vary both quantitatively and qualitatively with the parent species.

212

213 Our data suggests that hybridization is a more potent force for creating novel variation

214 than previously recognised, as many different combinations of the parental genomes

215 can arise in hybrids and allow for adaptive divergence between isolated populations

216 of the hybrid species. Importantly, we show that the variation is limited for DNA-

217 repair and mitochondrial genes. These may contribute to the general pattern of

218 reduced introgression on sex chromosomes, and are candidate loci for reproductive

219 loci that may be important in speciation. 


\section{$221 \quad$ Methods}

\section{$222 \quad$ Field sampling}

223 Italian sparrows were caught on Crete, Corsica and Sicily in 2013 and on Malta in

224 2014, while Spanish sparrows were caught in Lesina, Italy, 2008. House sparrows

225 were caught in Northern Norway between 2007 and 2013, and the outgroup tree

226 sparrow was caught in Sicily during 2008 (Supplementary Table 1). All sparrows

227 were caught using mist nets, and blood was sampled from the brachial vein and

228 immediately stored in Queens lysis buffer. Sparrows were released immediately after

229 blood sampling to minimize stress. All permits were obtained from appropriate

230 authorities prior to sampling.

232 Whole genome resequencing, data processing and analysis

\section{DNA extraction and sequencing}

234 DNA was extracted from blood samples stored in Queens lysis buffer using Qiagen

235 DNeasy Blood and Tissue Kits (Qiagen Corp., Valencia, CA), and stored in Qiagen

236 Elution Buffer (Qiagen Corp., Valencia, CA). Whole genome re-sequencing was

237 performed with Illumina sequencing technology. An Illumina TruSeq gDNA 180 bp

238 library was created and sequenced on the Illumina HiSeq 2000 platform with 100 bp

239 read length and three individuals per lane for the parent species, the tree sparrow and

240 the Italian sparrows from Malta; sparrows from Crete, Corsica and Sicily were

241 sequenced with four individuals per lane. All re-sequencing was performed by

242 Genome Quebec at McGill University (Montreal, Canada) 
243 (http://www.genomequebec.com/en/home.html). Raw data have been deposited at the

244 NCBI Sequence Read Archive under (Accessions YYYY).

\section{$245 \quad$ Variant calling and filtering}

246 All raw sequence reads were mapped to a repeat-masked version of the house sparrow 247 genome ${ }^{13}$ using BWA $0.7 .8^{31}$ with the mem, -M and - $\mathrm{R}$ options. A sorted BAM file 248 was produced using SAMTOOLS version $1.0^{32}$ using view with the $-\mathrm{b},-\mathrm{U}$ and $-\mathrm{s}$ 249 options and a pipe to the sort command. Duplicates were identified and filtered out 250 using MARKDUPLICATES from PICARD-TOOLS version 1.107

251 (http://broadinstitute.github.io/picard/) using the options validation stringency $=$ 252 lenient, assume sorted $=$ true and index $=$ true. Indels were identified using 253 RealignerTargetCreator and local realignments around these were performed with

254 IndelRealigner. Standard settings were used for both these tools which are 255 components of GATK 3.3.0 $0^{33,34}$. Final sequencing coverage of these final BAM files

256 (excluding duplicates) was 8x per individual (min. 5.99x, max. 15.8x; Supplementary 257 Table 2). Variants were then called using the GATK HaplotypeCaller. First, 258 HaplotypeCaller was run separately for each individual to create single-sample 259 gVCFs using the --emitRefConfidence GVCF, --variant_index_type LINEAR and -260 variant_index_parameter 128000 options, and then GATK GenotypeGVCFs tool was 261 run using standard settings to achieve joint genotyping. Two different versions of the 262 VCF file were created one with variable sites only (49 237560 SNPs), and one where 263 all sites were called as specified by the --includeNonVariantSites option (1 040518 264317 SNPs).

266 For both VCF files, indels were first filtered out using VCFtools version $0.1 .12 b^{35}$, 
267 and hard filtering according to the Broad Institute's recommendations was performed

268 with bcftools- $1.2^{32}$ in order to filter out sequencing artefacts. This included requiring

269 a QualByDepth of at least 2.0 and a FisherStrand phred-scaled p-value of less than

27060.0 , based on Fisher's Exact Test to detect strand bias in the reads, which may be

271 indicative of false positive calls. Hard filtering also required a RMSMappingQuality

272 of at least 40.0 to ensure high mapping quality of the reads across all samples, a

273 MappingQualityRankSumTest value of -12.5 to exclude reads where the alternate

274 alleles has a lower mapping quality than reads with the reference allele, and finally a

275 ReadPosRankSumTest value of less than -8.0 was required to ensure that reads with

276 the alternate allele were not shorter those with the reference allele, potentially

277 indicating sequencing artefacts. In addition, we filtered out sites with mean number of

278 reads per individual lower than 3, with a genotype quality lower than 20 and with a

279 mapping quality lower than 20 using VCFtools version $0.1 .12 \mathrm{~b}^{35}$. To avoid paralogs,

280 we also excluded sites with read depths above 5 times the variance of coverage. This

281 left a total of 38341426 sites for further analysis.

\section{PCA}

283 Principal component analysis was performed using $\mathrm{ANGSD}^{36}$ version 0.911 and

284 ngsTools version 1.0.1 $1^{37}$. This pipeline was chosen because it does not rely on

285 genotype calls but instead takes allele frequency likelihoods and genotype

286 probabilities into account ${ }^{38}$. We first estimated genotype probabilities from BAM files

287 with ANGSD, including bi-allelic sites only and allowing a minimum mapping and

288 site quality of 20 (Phred score) and a minimum coverage of 30x across all individuals.

289 PCA was then performed using ngsTools on genotype probabilities. Allele

290 frequencies were normalized and genotypes were not explicitly called, as specified by

291 setting the options -norm 0 and -call 0 options. Eigenvalues for each PC were then 
estimated from the covariance matrix produced by ngsTools and data was plotted using a custom R script. We used the broken stick criteria to assess which PC axes covariance from these axes (Supplementary Table 3). The analysis was performed on

296 three datasets: all sites, sites from the Z chromosome only, and autosomal sites only.

\section{Population genomic analysis}

298 Population genetic parameters were estimated for non-overlapping $100 \mathrm{~kb}$ windows

299 along the genome, as this window size was larger than the distance of LD-decay ${ }^{13}$.

300 Population genetic inference was based on genotype likelihoods whenever possible.

301 ANGSD $^{36}$ version 0.911 was used to estimate allele frequency likelihoods and to

302 obtain a maximum likelihood estimate of the unfolded site frequency spectrum (SFS)

303 for estimation of Tajima's D. Nucleotide diversity was estimated by dividing the

304 pairwise Theta (population scaled mutation rate) estimates by the number of variable

305 sites per window. The ancestral sequence was reconstructed using genotypes from the

306 outgroup tree sparrow. A Fasta file of the tree sparrow genome was obtained by using

307 the -doFasta 2 command with the -GL 1 -doCounts 1, -setMinDepth 3 and -

308 setMaxDepth 65 options in ANGSD $^{36}$ version 0.911. Here, BAM files from eight

309 additional tree sparrows, sequenced to 8-12x depth and processed as described for the

310 other samples above, were used. Genetic differentiation $\left(F_{\mathrm{ST}}\right)$ based on genotype

311 likelihood was estimated based on the two-dimensional SFS using ngsTools ${ }^{38}$. All

312 these analyses were performed on the final BAM files. Sequence divergence $\left(d_{\mathrm{xy}}\right)$ was

313 calculated from the VCF file with all positions called using the script developed in $^{39}$

314 (https://github.com/johnomics/Martin_Davey_Jiggins_evaluating_introgression_statis

315 tics/blob/master/egglib_sliding windows.py; version August 2014). 


\section{Admixture analysis}

317 Genetic admixture was estimated using ADMIXTURE ${ }^{40}$ version 0.911. The VCF-file

318 was converted to plink's PED format using VCFtools version $0.1 .12 b^{35}$ and plink

319 version $1.07^{41}$. Log likelihood values for $\mathrm{K}$, the number of genetic clusters in the

320 datasets, between $K=1$ and $K=8$ were estimated (Supplementary Table 4), and

321 admixture analyses were run for the most appropriate value of K. Analyses were first

322 run for a LD-pruned whole genome dataset (sites within a 50 SNP stepping window

323 with a correlation coefficient higher than 0.1 were omitted; pruning of the BED file

324 was performed with plink version 1.07 using the --indep-pairwise command; this left

325438,443 sites for analysis). Sliding window analyses with $100 \mathrm{~kb}$ windows were then

326 carried out to investigate variability in probability of parental inheritance across the

327 genome. This analysis was performed individually for each island population together

328 with the two parent species. The VCF-file was then split into individual VCF files per

$329100 \mathrm{~kb}$ using the $-\mathrm{L}$ option in GATK $3 \cdot 3 \cdot 0^{33,34}$. Individual ADMIXTURE analyses for

$330 \mathrm{~K}=2$ were then performed for each $100 \mathrm{~kb}$ BED file. A mean estimated cluster

331 assignment probability for all individuals per population was computed for each

332 analysis using a custom python script.

\section{Phylogenetic analyses}

334 To investigate whether phylogenetic relationships varied across the genome due to

335 introgression or incomplete lineage sorting, a machine-learning approach

336 implemented in SAGUARO version $0.1^{42}$ was used to identify genomic regions

337 characterized by distinct similarity matrices. To focus on breakpoints at which

338 recombination led to changes in the topology of populations or species, rather than

339 topological changes within populations, one representative high-coverage individual

340 per Italian sparrow population or sparrow species was used in these analyses. These 
341 were the house sparrow 8L19786, the Spanish sparrow Lesina_280 and the Italian sparrows C081 (Crete), K035 (Corsica), S059 (Sicily) and M036 (Malta). The

343 program VCF2HMMFeature (included in the SAGUARO package) was used to

344 convert the VCF-file to the HMMFeature format required by SAGUARO, and

345 SAGUARO was run using default parameters. Analysis was performed jointly for all

346 chromosomes, as the same similarity matrices are expected to occur on multiple

347 chromosomes. A total of 797 contiguous regions of up to 54,398 bp were identified

348 and assigned to one out of 41 similarity matrices, of which the most common

349 similarity matrix characterized $79.08 \%$ of the genome. Similarity matrices represented

350 by more than $2 \%$ of the genome are depicted in Figure 1.

351 The genomic regions identified by SAGUARO were subsequently used to infer

352 differences in phylogenetic relationships more thoroughly with the Bayesian software

353 BEAST version 2.2.0 $0^{43}$. To this end, chromosome-length alignments were first phased

354 using SHAPE-IT version $2^{44}$. To improve phasing, this analysis was conducted with a

355 subset consisting of the six individuals with the highest coverage per population (24

356 individuals in total) rather than just the six individuals used for SAGUARO analyses,

357 however, the six focal individuals were extracted from the alignments following

358 phasing. The phased chromosome-length alignments were then used to extract 38,964

359 non-overlapping blocks of $25,000 \mathrm{bp}$ from the 797 contiguous regions identified with

360 SAGUARO. For each of the 38,964 blocks, one of the two phased sequences per

361 individual was excluded at random, so that each alignment contained a single

362 sequence per population or species. To identify alignments particularly suitable for

363 Bayesian phylogenetic analysis, we quantified, for each alignment, the proportion of

364 missing data, the number of parsimony-informative sites, the proportion of

365 heterozygous sites, the mean bootstrap support of maximum-likelihood trees 
366 generated with RAXML version $8.2 .4^{45}$, and the probability that the alignment is free

367 of recombination determined with the Phi test ${ }^{46}$. We assumed that alignments with a

368 low proportion of heterozygous sites are less likely to contain paralogous sequences,

369 and that alignments with many parsimony-informative sites and high mean bootstrap

370 support contain strong phylogenetic signal. Thus, alignments were selected according

371 to the following "relaxed" and "strict" filters: a proportion of missing data below 0.2

372 (relaxed) or 0.1 (strict), at least 75 (relaxed) or 100 (strict) parsimony-informative

373 sites, a proportion of heterozygous sites below 0.005 (relaxed) or 0.0025 (strict), a

374 mean bootstrap support of at least 90 (both relaxed and strict), and a Phi test $p$-value

375 above 0.005 (relaxed) or 0.01 (strict). A total of 1,234 and 116 alignments were

376 selected with these relaxed and strict filters, respectively. To include an outgroup for

377 phylogenetic analyses with BEAST, consensus sequences of tree sparrow reads from

378 the Naxos1 individual mapped to the house sparrow reference genome ${ }^{13}$ were added

379 to each selected alignment. To avoid bias towards the reference, missing data were

380 not replaced by the reference alleles. The phylogeny of each alignment was then

381 inferred with BEAST, using the GTR model of sequence evolution with estimated

382 base frequencies, a Yule tree prior ${ }^{47}$, and 50 million Markov chain Monte Carlo

383 iterations. The ingroup, combining house sparrow, Spanish sparrow, and the

384 representatives of Italian sparrow populations was constrained to be monophyletic. In

385 the absence of a reliable absolute time line for sparrow divergences, the time of

386 divergence of ingroup and outgroup was fixed at 1 time unit, so that all divergence

387 ages within the ingroup are estimated relative to this initial split. Convergence of all

388 MCMC chains was confirmed by effective samples sizes greater than 500 for all

389 model parameters. 
391 Presence of introgression was estimated using Patterson's D ${ }^{48,49}$ calculations, using

392 the scripts provided in ${ }^{39}$. ABBA-BABA estimates were calculated using a minimum

393 coverage of 3 , a $100 \mathrm{~kb}$ window size and 1000 informative sites using the

394 egglib_sliding_windows.py script. The test was set up to estimate Spanish sparrow

395 introgression into a house sparrow background, with tree sparrow as an outgroup.

\section{Mitochondrial DNA}

397 Mitochondrial DNA gvcfs were called separately with haploid settings using the

398 -ploidy argument in HaplotypeCaller, jointly genotyped, and filtered as described

399 above using GATK 3.3.0 $0^{33,34}$. Fitchi version $1.1 .4^{50}$ was used to reconstruct a

400 haplotype genealogy based on Fitch distances.

\section{Recombination rate and common differentiation}

402 Genome-wide recombination rates were estimated using a house sparrow linkage

403 map. As the recombination map was produced using SNP chip data, recombination

404 distance estimates were first averaged using a sliding window approach and then a

405 loess fit of mean recombination rate against physical distance was performed in order

406 to interpolate fine scale variation across non-overlapping $100 \mathrm{~kb}$ windows. Since

407 recombination data were not available for the $\mathrm{Z}$ chromosome, this interpolation was

408 performed on autosomes only.

409 To test whether there was a relationship between recombination rate variation and

410 relative genomic differentiation, either $F_{\mathrm{ST}}$ from $100 \mathrm{~kb}$ non-overlapping windows,

411 which is a direct measure of relative differentiation, or the common differentiation

412 axis - i.e. shared differentiation amongst groups of populations - were used. The

413 latter was calculated by performing PCA on multiple pairwise comparisons of

414 differentiation featuring the same focal species following ${ }^{51}$. Common differentiation 
was estimated amongst 1) all Italian populations, 2) between all Italian populations

416 and the house sparrow and 3) between all Italian populations and the Spanish

417 sparrow. In each case, all pairwise $F_{\mathrm{ST}}$ comparisons including these species were

418 included, and the first principal component extracted.

\section{Outlier gene analysis}

420 Disparities in $F_{\mathrm{ST}}$ values between lineages were used to identify genomic regions in

421 which the Italian sparrow populations display elevated divergence from either or both

422 of their parents or other Italian sparrow populations. This approach is reminiscent to

423 population branch statistics. Three categories of outliers were of interest: Between

424 island outliers, where island populations differed strongly in parental resemblance,

425 were selected as these are informative of how Italian sparrow populations are

426 differentially adapted. Private outliers, windows in which Italian sparrows are

427 diverged from both parent species, show where unique adaptation is putatively

428 strongly selected for. Finally, portions of the genome invariably inherited from the

429 same parent species for all populations are informative of parts that are under strong

430 selection to resemble a specific parent species and may reveal constraints on hybrid

431 speciation.

432 Between island outliers:

433 The $1 \%$ of $100 \mathrm{~kb}$ regions which differed most with respect to $F_{\mathrm{ST}}$ against the parental

434 species between two islands were selected for all possible island-island combinations.

435 All genes within or partly within these regions were then extracted. As historical

436 effects such as ancestral polymorphism and selection prior to the parental split can be

437 assumed to be constant across Italian populations, using the difference in $F_{\text {ST }}$ against

438 the same parent species will yield results which are not dependent on these factors. 
439 Furthermore, $F_{\text {ST }}$ was not strongly dependent on recombination rate between Italian 440 sparrow populations (Supplementary Fig. 4).

\section{Private Outliers:}

442 Outliers in which Italian sparrows were differentiated from both parent species,

443 hereafter private outliers, were extracted from the $1 \%$ of windows exhibiting the

444 largest difference in $F_{\mathrm{ST}}$ between each hybrid/parent comparison, only keeping

445 windows overlapping between both hybrid/parent comparisons. This was done

446 separately for each Italian population, and all outliers detected across the populations

447 were then merged for gene ontology analyses.

448 Outliers invariably resembling one parent species:

449 To limit historical effects, due to for instance ancestral polymorphism and selection

450 prior to the parental split, we used the $100 \mathrm{~kb}$ windows in which the Italian sparrow

451 had the cumulative largest difference in $F_{\mathrm{ST}}$ value between one parent and the other.

452 This was achieved by summing the $F_{\text {ST }}$ values between all Italian sparrow populations

453 and house sparrow, and subtract the sum of the $F_{\mathrm{ST}}$ values between these populations

454 and Spanish sparrow. As the resulting distribution was skewed, using a percentage at

455 each tail would not have captured the biological pattern where house sparrow

456 inheritance across populations was more common than Spanish sparrow inheritance

457 across all populations. Therefore, we squared the summed values and extracted the

$4582 \%$ of the windows that deviated most strongly from 0 (Supplementary Fig. 8B),

459 which yielded more invariably house sparrow like outliers than invariably Spanish

460 sparrow like outliers.

461 For all outlier windows in each of the three categories above, annotated genes that

462 resided completely or partially within them were extracted for separate gene ontology 
463 analysis. One analysis was performed on all private outliers identified across

464 populations, one on all outliers between populations, including all combinations of

465 populations, one on outliers that resembled house sparrow across all populations, and

466 finally one on outliers that resembled Spanish sparrow across all populations. As only

46714 outliers resembling Spanish sparrow across all populations were found, no

468 significant GO-terms were found for this analysis. Therefore, we do not provide a

469 table of significant terms for this analysis. These analyses were performed using GO-

470 stat $^{52}$, with a human reference base. We implemented standard settings for GO

471 analyses, i.e. a values of 3 as the minimal length of considered GO paths and no

472 merging of GOs if gene lists overlap. Mito-nuclear genes were identified using

473 MITOMINER $4.0^{53}$ with a human reference database and standard settings.

474 Overrepresentation of mitonuclear genes was subsequently tested using a Chi-square

475 test. Corrections for multiple testing were performed with the Benajmini method.

477 DN/DS analyses

478 To test if the $\mathrm{Z}$ chromosome was under stronger selection, synonymous and non-

479 synonymous fixed differences within genes against each parent species for all

480 autosomes and the $\mathrm{Z}$ chromosome were estimated for each Italian sparrow population.

481 A goodness of fit test was applied to test if the number of nonsynonymous

482 substitutions on the $\mathrm{Z}$ chromosome was higher than expected for each parent species.

483 To this end, the R package PopGenome ${ }^{54}$ was used. The splitting,data command was

484 used to extract genes and fixed sites were extracted. Synonymous and

485 nonsynonymous sites were then identified using the options subsites="syn" and

486 subsites="nonsyn", respectively. 


\section{Linkage disequilibrium decay}

489 To address whether linkage disequilibrium (LD) was higher and LD decayed more

490 slowly in outlier windows than in randomly selected windows, plink version

$4911.90 \mathrm{~b} 3 \mathrm{~b}^{41}$ was used. Using --const-fid --ld-window 1000 --ld-window-kb 100 --r2 and

492 -ld-window-r2 0.0 , linkage disequilibrium in the $100 \mathrm{~kb}$ outlier windows was

493 estimated within each of the Italian populations. In addition, we randomly selected

$4941,000100 \mathrm{~kb}$ windows spread across the chromosomes in proportion with

495 chromosome size and estimated linkage disequilibrium for these in the same manner

496 for comparison. A linear model was fitted for each outlier window, and intercept and

497 slope were recorded and used in glm's to test whether LD was higher and LD decay

498 was slower in outlier windows than in randomly selected windows. 


\section{References}

500 1. Mallet, J. Hybridization as an invasion of the genome. Trends Ecol. Evol. 20, $501 \quad 229-237(2005)$.

502 2. Abbott, R. et al. Hybridization and speciation. J. Evol. Biol. 26, 229-246

503 (2013).

504 3. Seehausen, O. Hybridization and adaptive radiation. Trends Ecol. Evol. 19, $505 \quad 198-207$ (2004).

506 4. The Heliconius Genome Sequencing Consortium. Butterfly genome reveals

507 promiscuous exchange of mimicry adaptations among species. Nature 487, 94-98

508 (2012).

$5095 . \quad$ Rieseberg, L. H. Major Ecological Transitions in Wild Sunflowers Facilitated 510 by Hybridization. Science 301, 1211-1216 (2003).

511 6. Hermansen, J. S. et al. Hybrid speciation in sparrows I: phenotypic

512 intermediacy, genetic admixture and barriers to gene flow. Mol. Ecol. 20, 3812-3822 513 (2011).

$5147 . \quad$ Trier, C. N., Hermansen, J. S., Sætre, G.-P. \& Bailey, R. I. Evidence for Mito-

515 Nuclear and Sex-Linked Reproductive Barriers between the Hybrid Italian Sparrow

516 and Its Parent Species. PLoS Genet 10, e1004075-10 (2014).

517 8. Sankararaman, S. et al. The genomic landscape of Neanderthal ancestry in 518 present-day humans. Nature 507, 354-357 (2014).

519 9. Fontaine, M. C. et al. Extensive introgression in a malaria vector species 520 complex revealed by phylogenomics. Science 347, 1258524-1258524 (2015).

521 10. Baack, E. J. \& Rieseberg, L. H. A genomic view of introgression and hybrid 522 speciation. Curr. Opin. Genet. Dev. 17, 513-518 (2007). 
523 11. Martin, S. H. et al. Genome-wide evidence for speciation with gene flow in

524 Heliconius butterflies. Genome Res. 23, 1817-1828 (2013).

525 12. Qvarnström, A. \& Bailey, R. I. Speciation through evolution of sex-linked

526 genes. Heredity 102, 4-15 (2008).

527 13. Elgvin, T. O. et al. The genomic mosaicism of hybrid speciation. In review,

528 Science Advances. MS attached in submission.

529 14. Saetre, G. P. et al. Single origin of human commensalism in the house

530 sparrow. J. Evol. Biol. 25, 788-796 (2012).

531 15. Bache-Mathiesen, L. The Evolutionary Potential of Male Plumage Color in a

532 Hybrid Sparrow Species. UiO DUO vitenarkiv

533 https://www.duo.uio.no/handle/10852/45473

534 16. Burri, R. et al. Linked selection and recombination rate variation drive the

535 evolution of the genomic landscape of differentiation across the speciation continuum

536 of Ficedula flycatchers. Genome Res. 25, 1656-1665 (2015).

537 17. Hill, W. G. \& Robertson, A. The effect of linkage on limits to artificial

538 selection. Genet. Res. 8, 269-294 (1966).

539 18. Laine, V. N. et al. Evolutionary signals of selection on cognition from the

540 great tit genome and methylome. Nat. Commun. 7, 1-9 (2016).

541 19. Lamichhaney, S. et al. Evolution of Darwin's finches and their beaks revealed

542 by genome sequencing. Nature 518, 371-375 (2015).

543 20. Eroukhmanoff, F., Hermansen, J. S., Bailey, R. I., Sæther, S. A. \& Sætre, G.-

544 P. S. Local adaptation within a hybrid species. Heredity 111, 286-292 (2013).

545 21. Noramly, S., Freeman, A. \& Morgan, B. A. Beta-catenin signaling can initiate

546 feather bud development. Development 126, 3509-3521 (1999). 
547 22. Guo, H. et al. Wnt/beta-catenin signaling pathway activates melanocyte stem

548 cells in vitro and in vivo. J. Dermatol. Sci. 83, 45-51 (2016).

549 23. Mank, J. E., Nam, K. \& Ellegren, H. Faster-Z evolution is predominantly due 550 to genetic drift. Mol. Biol. Evol. 27, 661-670 (2010).

551 24. Charlesworth, B., Coyne, J. A. \& Barton, N. H. The Relative Rates of

552 Evolution of Sex Chromosomes and Autosomes. Am. Nat. 130, 113-146 (2016).

553 25. David, W. M., Mitchell, D. L. \& Walter, R. B. DNA repair in hybrid fish of

554 the genus Xiphophorus. Comp. Biochem. Physiol. C Toxicol. Pharmacol. 138, 301$555309(2004)$.

556 26. Greig, D., Travisano, M., Louis, E. J. \& Borts, R. H. A role for the mismatch 557 repair system during incipient speciation in Saccharomyces. J. Evol. Biol. 16, 429$558437(2003)$.

559 27. Schumer, M. \& Brandvain, Y. Determining epistatic selection in admixed 560 populations. Mol. Ecol. 25, 2577-2591 (2016).

561 28. Barton, N. H. The role of hybridization in evolution. Mol. Ecol. 10, 551-568 562 (2001).

563 29. Poelstra, J. W., Vijay, N., Hoeppner, M. P. \& Wolf, J. B. W. Transcriptomics 564 of colour patterning and coloration shifts in crows. Mol. Ecol. 24, 4617-4628 (2015).

565 30. Tomarev, S. I. \& Nakaya, N. Olfactomedin Domain-Containing Proteins:

566 Possible Mechanisms of Action and Functions in Normal Development and

567 Pathology. Mol. Neurobiol. 40, 122-138 (2009). 


\section{Additional References for Methods Section}

569 31. Li, H. \& Durbin, R. Fast and Accurate Short Read Alignment with Burrows-

570 Wheeler Transform. Bioinformatics 25, 1754-1760 (2009).

571 32. Li, H. et al. The Sequence Alignment/Map format and SAMtools.

572 Bioinformatics 25, 2078-2079 (2009).

573 33. McKenna, A. et al. The Genome Analysis Toolkit: A MapReduce framework

574 for analyzing next-generation DNA sequencing data. Genome Res. 20, 1297-1303

575 (2010).

576 34. Van der Auwera, G. A. et al. From FastQ Data to High-Confidence Variant

577 Calls: The Genome Analysis Toolkit Best Practices Pipeline. Curr. Protoc.

578 Bioinformatics. 11, 1-33 (2013).

579 35. Danecek, P. et al. The variant call format and VCFtools. Bioinformatics 27, $580 \quad 2156-2158(2011)$.

581 36. Korneliussen, T. S., Albrechtsen, A. \& Nielsen, R. ANGSD: Analysis of Next

582 Generation Sequencing Data. BMC Bioinformatics 15, 443-13 (2014).

583 37. Fumagalli, M., Vieira, F. G., Linderoth, T. \& Nielsen, R. ngsTools: methods

584 for population genetics analyses from next-generation sequencing data.

585 Bioinformatics 30, 1486-1487 (2014).

586 38. Fumagalli, M. et al. Quantifying Population Genetic Differentiation from

587 Next-Generation Sequencing Data. Genetics 195, 979-992 (2013).

588 39. Martin, S. H., Davey, J. W. \& Jiggins, C. D. Evaluating the Use of ABBA-

589 BABA Statistics to Locate Introgressed Loci. Mol. Biol. Evol. 32, 244-257 (2014).

590 40. Alexander, D. H., Novembre, J. \& Lange, K. Fast model-based estimation of 591 ancestry in unrelated individuals. Genome Res. 19, 1655-1664 (2009). 
594 42. Zamani, N. et al. Unsupervised genome-wide recognition of local relationship 595 patterns. BMC Genomics 14, 1-11 (2013).

596 43. Bouckaert, R. et al. BEAST 2: A Software Platform for Bayesian Evolutionary 597 Analysis. PLoS Comput. Biol. 10, e1003537-6 (2014).

598 44. O'Connell, J. et al. A General Approach for Haplotype Phasing across the Full 599 Spectrum of Relatedness. PLoS Genet. 10, e1004234-21 (2014).

600 45. Stamatakis, A. RAxML version 8: a tool for phylogenetic analysis and post601 analysis of large phylogenies. Bioinformatics 30, 1312-1313 (2014).

602 46. Bruen, T. C., Philippe, H. \& Bryant, D. A simple and robust statistical test for 603 detecting the presence of recombination. Genetics 172, 2665-2681 (2006).

604 47. Yule, U. G. A Mathematical theory of evolution, based on the conclusions of 605 Dr. J. C. Willis, F.R.S. Philos. Trans. R. Soc. Lond. B. Biol. Sci. 213, 21-87 (1925).

606 48. Green, R. E. et al. A draft sequence of the Neandertal genome. Science 328, $607 \quad 710-722(2010)$.

608 49. Durand, E. Y., Patterson, N., Reich, D. \& Slatkin, M. Testing for Ancient 609 Admixture between Closely Related Populations. Mol. Biol. Evol. 28, 2239-2252 610 (2011).

611 50. Matschiner, M. Fitchi: haplotype genealogy graphs based on the Fitch 612 algorithm. Bioinformatics 32, 1250-1252 (2016).

613 51. Burri, R. et al. Linked selection and recombination rate variation drive the 614 evolution of the genomic landscape of differentiation across the speciation continuum 615 of Ficedulaflycatchers. Genome Res. 25, 1656-1665 (2015). 
616 52. Beissbarth, T. \& Speed, T. P. GOstat: find statistically overrepresented Gene

617 Ontologies within a group of genes. Bioinformatics 20, 1464-1465 (2004).

618 53. Smith, A. C., Blackshaw, J. A. \& Robinson, A. J. MitoMiner: a data

619 warehouse for mitochondrial proteomics data. Nucleic Acids Res. 40, D1160-D1167

620 (2011).

621 54. Pfeifer, B., Wittelsbürger, U., Ramos-Onsins, S. E. \& Lercher, M. J.

622 PopGenome: an efficient Swiss army knife for population genomic analyses in R.

623 Mol. Biol. Evol. 31, 1929-1936 (2014). 
625 Supplementary Information is linked to the online version of the paper at www.nature.com/nature.

\section{Acknowledgements}

629 We thank Maria Tesaker and BirdLife Malta for help with field work, Laura Piñeiro 630 and Lena Bache-Mathiesen for providing morphological data, and Anna Nilsson for 631 comments on the manuscript. This work was funded by a Swedish Research Council 632 post doctoral grant and a Wenner-Gren Fellowship to A.R. and a Norwegian Science 633 Foundation grant to G-P.S. and A.R.

\section{Author Contributions}

636 A.R. conceived the study, carried out field work, lab work, designed analyses, 637 analysed data and wrote the manuscript. C.N.T. helped design analyses, and provided 638 example scripts for many analyses, F.E. carried out field work and the gene ontology 639 analyses, J.S.H. carried out field work and the final touches in figure preparation, 640 M.M. did the BEAST and Saguaro analyses and M.R. performed the recombination 641 rate analyses and PCA. T.E. provided the house sparrow reference genome, and

642 G.P.S. identified the study system, designed the sampling strategy and carried out

643 field work. All co-authors commented on the manuscript.

645 Data is deposited at YYYY. Reprints and permissions information is available at

646 www.nature.com/reprints. We declare no competing financial interests.

647 Correspondence and requests for materials should be addressed to

648 anna.runemark@ibv.uio.no 

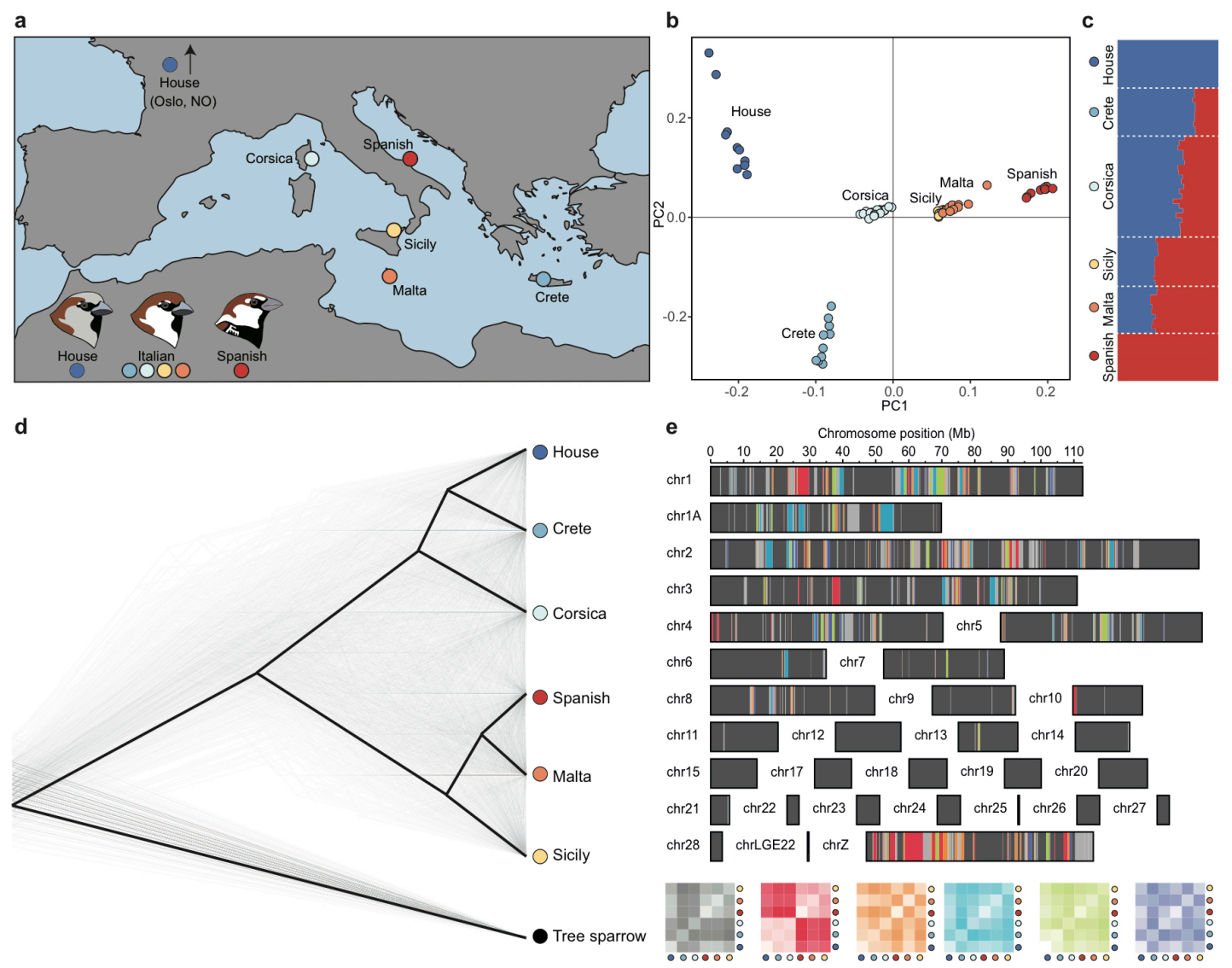

f

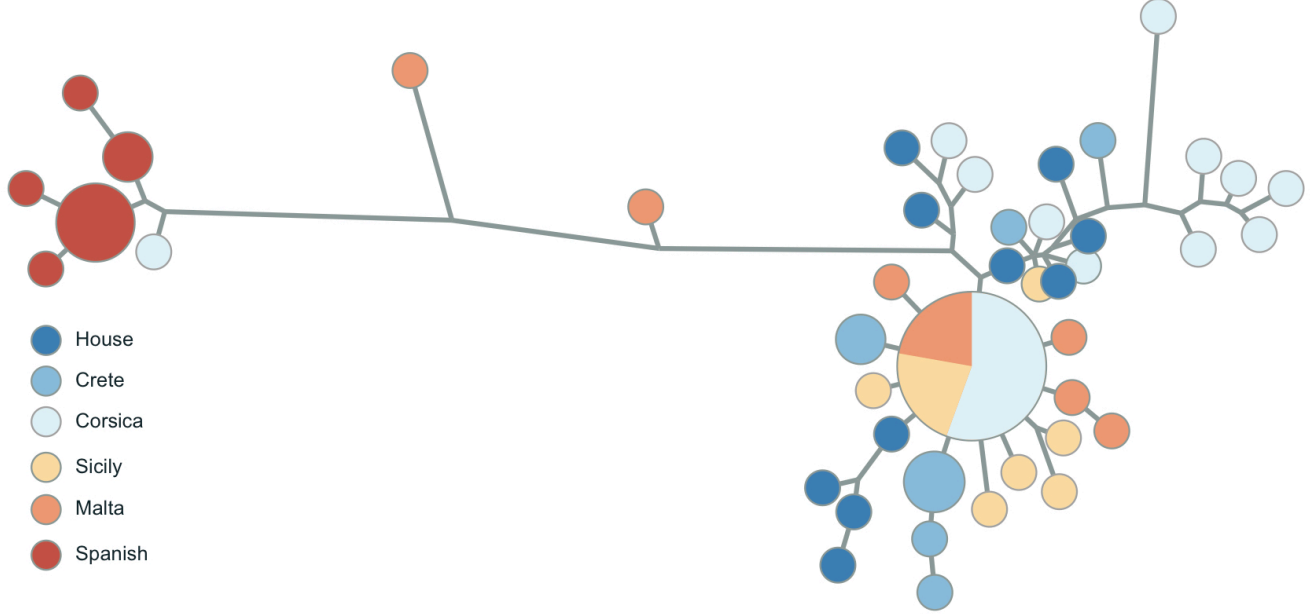

Figure 1. Population structuring of the focal taxa. a, Map showing the location of

651 the island populations and the reference parent species populations, with examples of male plumage patterns. b, PCA of LD-pruned high quality SNP set with eigenvector 1

653 on the $\mathrm{x}$-axis and eigenvector 2 on the $\mathrm{y}$-axis. c, Population structuring based on

654 Admixture analysis for house sparrow, Italian and Spanish sparrow populations. d,

655 BEAST trees illustrating genome-wide variation in phylogenetic clustering between

656 the taxa. e, SAGUARO plot illustrating the distribution of the six most common

657 relatedness matrixes over the genome. f, Haplotype genealogy graph of mitochondrial sequences. 

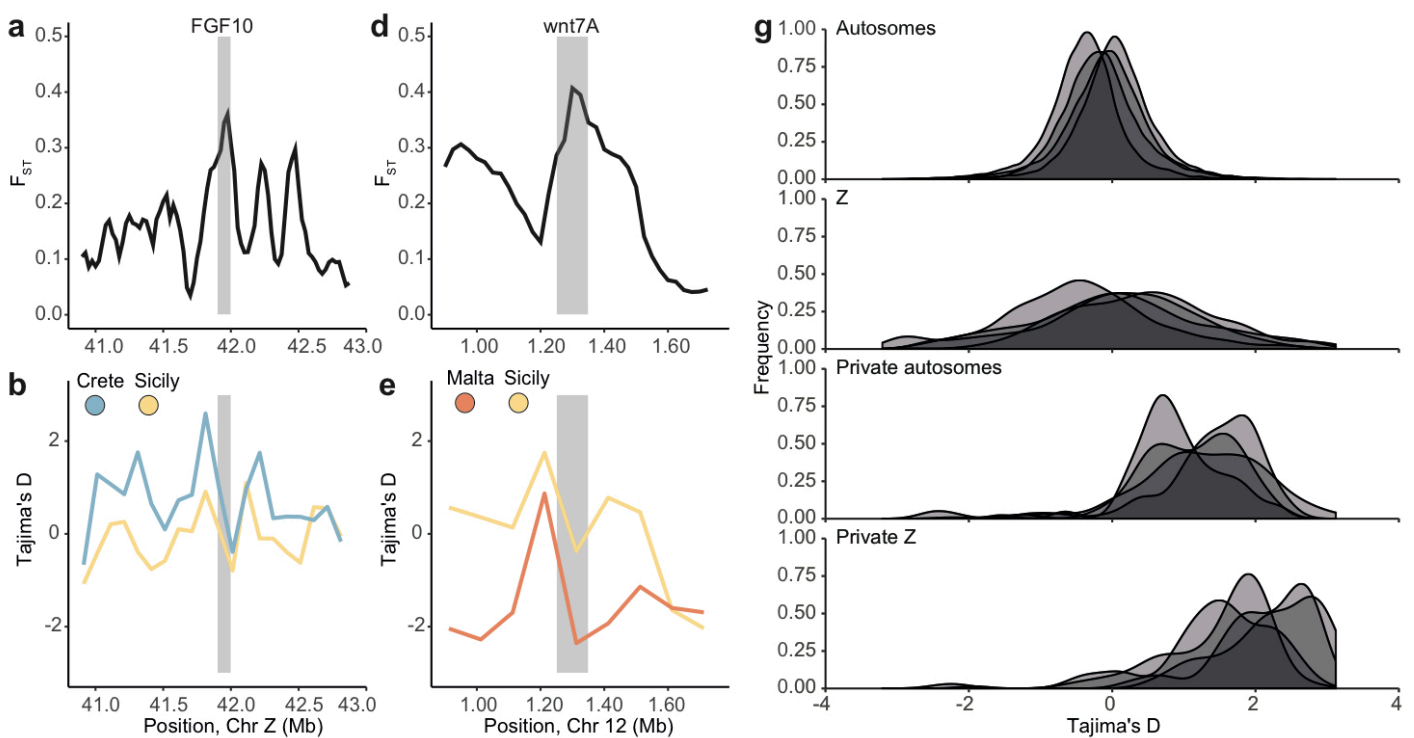

c
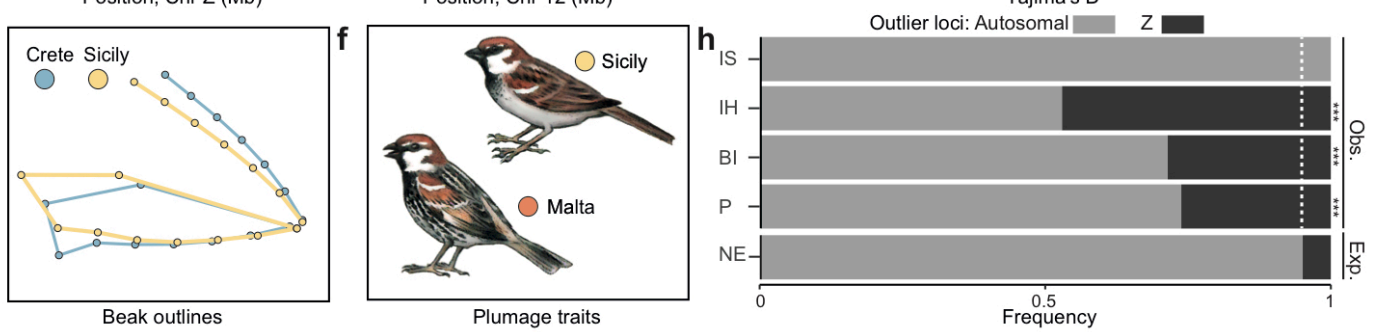

660 Figure 2. Local adaptation, private variation, and strong selection on the Z-

661 chromosome. a, The beak shape candidate gene FGF10 is differentiated between

662 Italian sparrows on Crete and Sicily. b, Tajima's D for the FGF10 region. c, Beak

663 shape differences between Cretan and Sicilian sparrows. d, The plumage candidate

664 gene wnt7a is differentiated between genetically similar sparrow populations on

665 Sicily and Malta. e, Tajima's D for the wnt7a region. f, Schematic illustration of

666 plumage differences between the populations. g, Strength of selection on the

667 autosomes, the Z-chromosome and on private outlier loci where the Italian sparrow is

668 differentiated from both parent species. $\mathbf{h}$, Distribution of outlier genes between the

669 Z-chromosome and the autosomes. IS denotes invariably Spanish, IH invariably

670 house, BI between islands, P private and NE neutral expectations. 


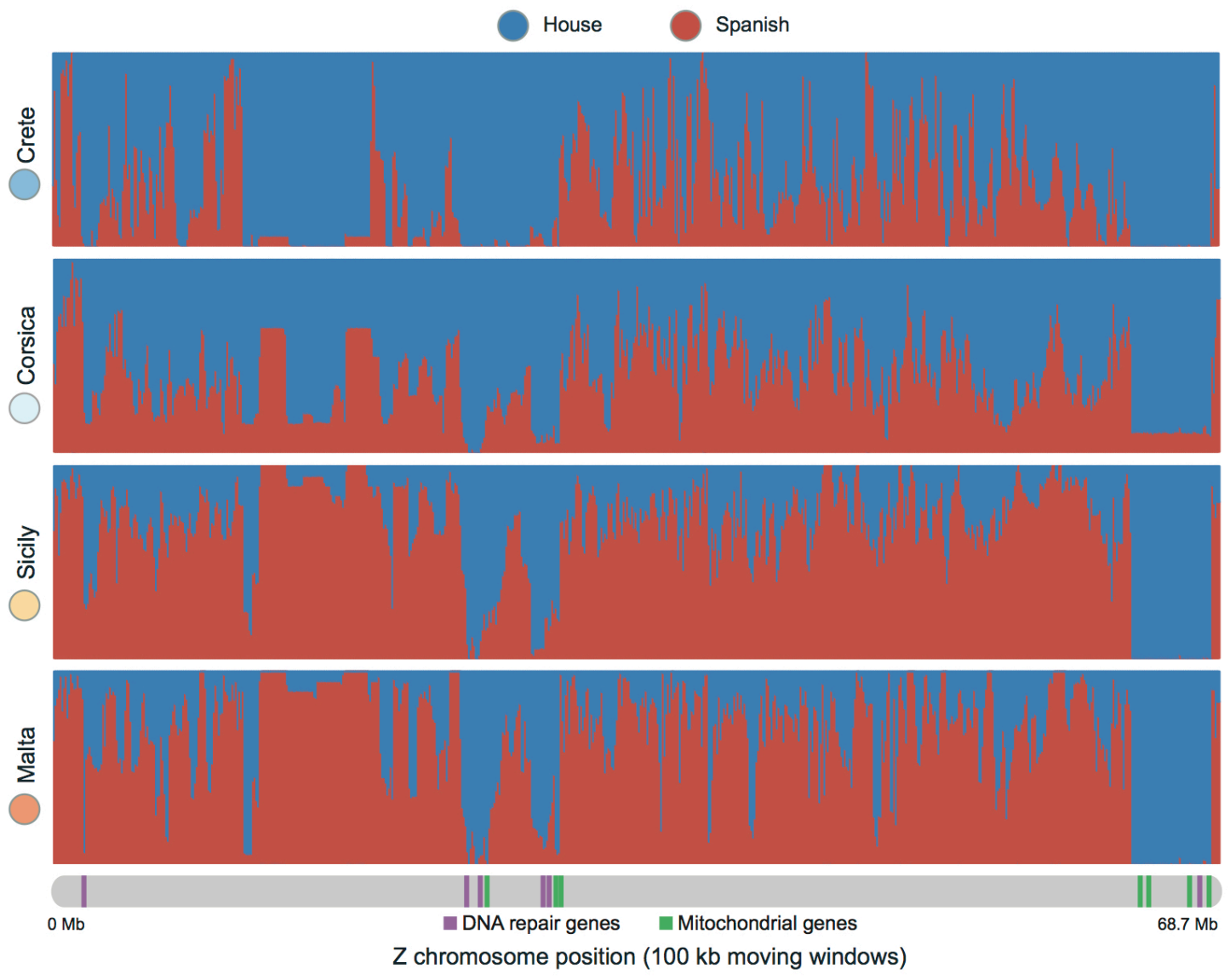

672 Figure 3. Parental similarity across the Z-chromosome. Sliding window

673 ADMIXTURE analysis of probability of house sparrow (blue) or Spanish sparrow

674 (red) inheritance over the Z-chromosome for the four Italian sparrow populations.

675 Areas highly constrained to house sparrow inheritance harbour a significantly higher

676 proportion DNA repair genes (green lines) and many mito-nuclear (purple lines)

677 genes. 